\title{
Evolución de los parámetros geométricos de diseño en puentes construidos con voladizos sucesivos in situ
}

\section{Evolution of geometrical design parameters for cast in place bridges built using the cantilever method}

\author{
Ruben Valle - Pascual $^{1}$, Nestor Carvajal - Monsalve ${ }^{2}$, Juan Carlos Botero - Palacio ${ }^{3}$ \\ ${ }^{1}$ Mecánica aplicada, Departamento de ingeniería civil, Universidad EAFIT, Colombia. Email: rvallep@ eafit.edu.co \\ ${ }^{2}$ Estructuras \& Sísmica, Colombia. Email: nfcarvajal@gmail.com \\ ${ }^{3}$ Mecánica aplicada, Departamento de ingeniería civil, Universidad EAFIT, Colombia. Email: jcbotero@eafit.edu.co
}

Recibido: Noviembre 10, 2016 Aceptado: Noviembre 30, 2016 Versión Final: Diciembre 30, 2016

\begin{abstract}
RESUMEN
Se estudiaron puentes construidos con voladizos sucesivos in situ, en donde se evalúan y se comparan las teorías utilizadas para su predimensionamiento desde el 1978 hasta la actualidad, así como la evolución de su diseño. Se recopilaron los datos obtenidos de las secciones transversales y longitudinales de 124 puentes construidos en voladizos sucesivos in situ en 29 países y posteriormente fueron evaluados. Estos 124 puentes son condensados en tres grupos: por cronología, por luz libre y por países. Estos datos son tratados estadísticamente y se comparan con los valores clásicos empleados en el diseño. El análisis de resultados permite obtener gráficas paramétricas asociadas a las condiciones de diseño. Las conclusiones muestran cómo varían los parámetros geométricos de diseño en el tiempo y como se van obteniendo valores óptimos que permiten diseñar y construir puentes más seguros y económicos.
\end{abstract}

PALABRAS CLAVE: Puentes, Voladizos sucesivos, Predimensionamiento, Análisis estadístico, Diseño óptimo.

\begin{abstract}
Cast-in-place bridges built using the cantilever method have been studied and evaluated. Also the theories used for their pre-dimensioning from 1978 to the present were compared, like the evolution of its design. The data were collected and obtained of transverse and longitudinal sections of 124 cast-in-place bridges built using the cantilever method in 29 countries and were subsequently evaluated. These 124 bridges are condensed into three groups by chronology, free span and by countries. These data are processed statistically and compared with classical values used in design. The analysis of results allows for parametric graphs associated with the design conditions. The conclusions shows how varying the geometric design parameters in time and how they will obtain optimal values that allows to design and build safer and economic bridges
\end{abstract}

KEYWORDS: Bridge, Balanced cantilever method, Pre-dimensioning, Statistically analysis, Optimal design.

\section{INTRODUCCIÓN}

El diseño y construcción de puentes ha ido evolucionando exponencialmente hasta la actualidad. A pesar de que son los parámetros subyacentes al proyecto de obra [1] [2] [3] [4] [5] [6] los que definen la metodología estructural a utilizar, aún se observa que es posible la elección entre varias tipologías estructurales y diseños para un mismo caso. Entre ellas se encuentra la construcción por voladizos sucesivos in situ, siendo esta la metodología abarcada en el presente estudio. Este método constructivo se define como [7] la ejecución del tablero del puente avanzando por tramos sucesivos, haciendo soportar a la parte ya construida el peso del tramo en construcción. Cada tramo o dovela se une y funde al precedente cuando adquiere una resistencia suficiente. Entonces se convierte en autoportante y sirve de base de arranque para un nuevo tramo. El primero que utilizó esta forma de diseño y construcción fue Freyssinet en 1928; sin embargo, fue Baumgart en 1930 quien

Este artículo puede compartirse bajo la licencia CC BY-ND 4.0 y se referencia usando el siguiente formato: R. Valle, N.F Carvajal, J.C Botero. "Evolución de los parámetros geométricos de diseño en puentes construidos con voladizos sucesivos in situ" UIS Ingenierías, vol. 16, no. 1, pp.85-100, Enero-Junio 2017. 
diseñó y construyó de esta manera el puente Herval en Brasil. En Colombia [2] el primer uso de esta tipología se da en el puente Currulao en Urabá (1962). En la figura 1 se muestran fotos del momento de su ejecución.
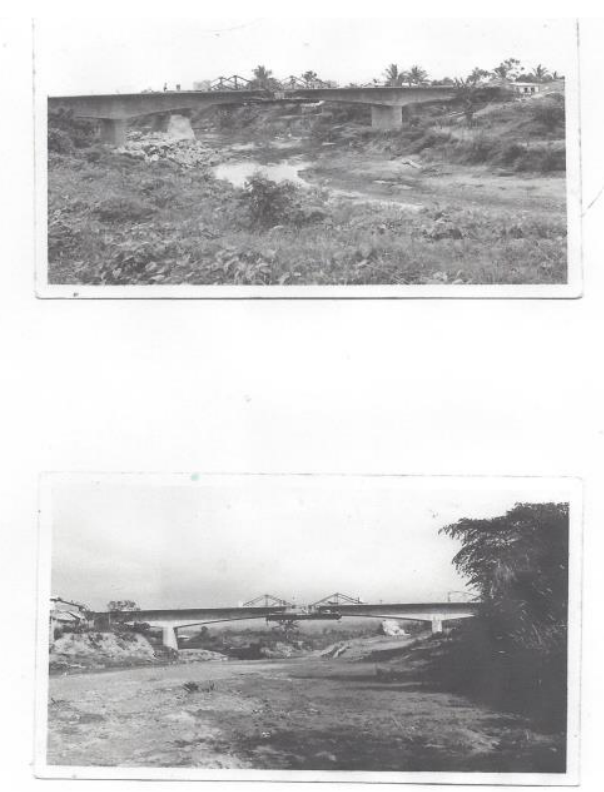

Figura 1. Ejecución del puente Currulao en Uraba (1962). Fuente. Ingeniero. Mario Rodriguez - Precomprimidos S.A

En el cálculo y diseño estructural de un puente, el proceso constructivo es algo inherente al modelo analítico, no puede estudiarse y tratarse uno de ellos sin considerar el otro. En el método de voladizos sucesivos no hay muchas posibilidades de variación de método en el proceso constructivo, pero no ocurre así en el diseño; donde se han dado variaciones significativas a lo largo de los años. Es por ello que en este estudio se comparan las distintas teorías de predimensionamiento utilizadas en las últimas décadas para entender la evolución de los puentes diseñados hasta hoy. También se trata la variación del proceso constructivo en la historia; que, aunque como se ha dicho es escasa, debe mencionarse.

\section{TEORÍAS DE PREDIMENSIONAMIENTO}

Se presentan 5 teorías [8][9][3] [10]11] sobre el predimensionamiento, de las que se van a exponer tanto sus semejanzas como sus diferencias. Mayoritariamente se centran en la sección transversal, pero también dan consejos para la sección longitudinal del puente. Estas teorías van desde el 1978 hasta la fecha.

En las figuras 2 y 3 se muestran los parámetros geométricos que se van a tratar en el estudio de las distintas teorías.

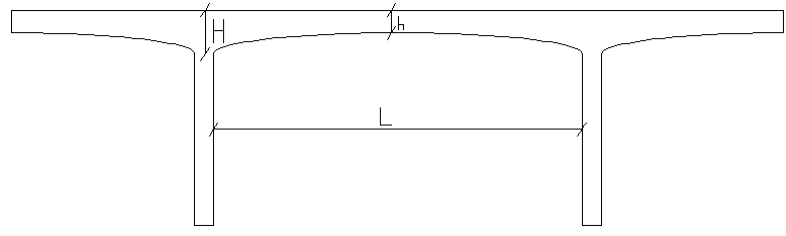

Figura 2. Vista de la sección longitudinal de un puente típico donde se muestran las variables estudiadas. Fuente. Elaboración propia.

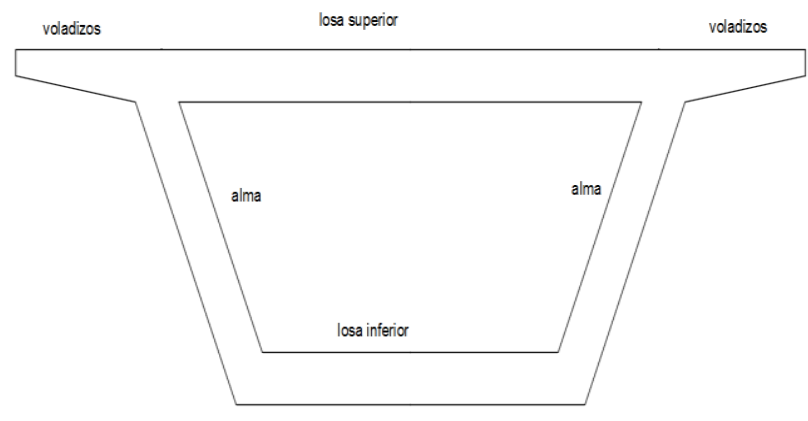

Figura 3. Vista de la sección transversal donde se muestran las variables estudiadas. Fuente. Elaboración propia.

\subsection{Precast segmental box girder manual (1978)}

Este documento elaborado por los institutos de pretensado y postensado de EE.UU (PTI y PCI) establece valores basados en la experiencia, pero da información limitada y generalizada. Los valores que recoge son relaciones y aproximaciones entre los parámetros mostrados en la figura 2 y la figura 3 . Estos valores se muestran a continuación:

- Relación H/L: $1 / 18$ - 1/20

- Relación h/L: $1 / 40$ - 1/50

- Voladizos: $1 / 4$ del ancho del tablero.

- Espesor losa superior: $17.5 \mathrm{~cm}-25 \mathrm{~cm}$

- Alma: $25 \mathrm{~cm}$ mínimo, recomendable $35 \mathrm{~cm}$

- Espesor losa inferior: Mínimo $17.8 \mathrm{~cm}$

\subsection{Construcción de puentes de hormigón pretensado por voladizos sucesivos. Mathivat. (1980)}

Esta teoría de predimensionamiento fue desarrollada por J. Mathivat (1980) y trata sobre la experiencia del autor. Establece algunas teorías elementales.

Entre ellas que es mejor pocas almas y anchas, que muchas y estrechas, ya que así se ahorra material; o la validez de cajones monocelulares para anchuras de tablero de hasta $12 \mathrm{~m}$. Se habla sobre cizalladura y sobre el acero de refuerzo. 


\subsection{Setra $(2003)$}

Fue desarrollado por un conjunto de ingenieros franceses y resume la historia de este tipo de puentes tanto en Francia como en el resto del mundo y además da directrices concretas sobre el diseño de estos. Particularmente trata cada elemento por separado (losa superior, losa inferior, almas, etc.) con sus respectivas recomendaciones para su diseño particular.

Aporta a todo lo tratado en las anteriores teorías el concepto de losa superior postensada, diseño del alma inclinada y comportamiento del postensado en el proceso constructivo.

\subsection{Puentes. Apuntes para su diseño, cálculo y construcción. Manterola (2006)}

Fue escrito por un ingeniero español [12] con más de 200 diseños de puentes. Ofrece teorías basadas en su experiencia.

Corrobora algunas de las teorías anteriores, como la de Mathivat. Contempla la opción de variar el espesor de la losa inferior; descendiendo este conforme se aleja de los pilares.

\subsection{Criterios para la optimización del} predimensionamiento de puentes en sección cajón. Ariñez (2012)

Es de los estudios más actualizados [11] de predimensionamiento de puentes de la tipología estudiada. Abarcan toda la información hasta el momento (incluyendo diferentes normas) y la procesan resultando, que las almas inclinadas son más eficientes que las rectas en cantos pequeños, y al contrario en las grandes.

Se hace un aporte muy importante, y es que establece que la relación óptima entre la luz (L) y el canto sobre pilares (H) es $1 / 17$, y que es mejor respetar este límite que aumentar espesores de los elementos.

\subsection{Comparativa de teorías de predimensionamiento}

Se procede a comparar las distintas teorías de predimensionamiento para unos mismos valores iniciales, que son los siguientes:

- Luz longitudinal $(\mathrm{L})=100 \mathrm{~m}$

- Ancho de tablero=12m

- $\mathrm{L} / \mathrm{H}=1 / 20$

- $\mathrm{L} / \mathrm{h}=1 / 50$

Operando según las distintas teorías de predimiensionamiento, se obtienen valores de:
- Espesor losa superior

- Espesor losa inferior

- Ancho de alma

- Separación entre almas (S)

- Espesor de voladizos

Además, se obtiene el área transversal asociada a los puntos donde se define $\mathrm{H}$ y h, la relación entre ellas, y el promedio de todas las teorías. También se analiza la relación entre estos resultados. Estos resultados se muestran en la tabla 1.

Tabla 1 Resumen de comparativa de teorías de predimensionamiento.

\begin{tabular}{|c|c|c|c|c|c|c|}
\hline $\mathrm{cm}$ & $\begin{array}{c}\text { Precast } \\
(1978) \\
\end{array}$ & $\begin{array}{c}\text { Mathivat } \\
\text { (1980) }\end{array}$ & $\begin{array}{l}\text { SETRA } \\
(2003) \\
\end{array}$ & \begin{tabular}{|c|} 
Manterola \\
$(2006)$
\end{tabular} & $\begin{array}{l}\text { Ariñez } \\
\text { (2012) }\end{array}$ & \\
\hline Losa sup & 24 & 22 & 24 & 28 & 30 & \\
\hline Losa inf & 20 & 20 & 20 & 22 & 22 & \\
\hline Almas & 30 & 27 & 26 & 28 & 27 & \\
\hline$S$ & 600 & 600 & 600 & 720 & 720 & \\
\hline Voladizos & - & - & 80 & 65 & 48 & Promedio \\
\hline $\begin{array}{c}\text { AREA en } \mathrm{H} \\
(\mathrm{m} 2)\end{array}$ & 5.45 & 5.08 & 5.79 & 6.82 & 7.03 & 6.034824 \\
\hline $\begin{array}{c}\text { AREA en } \mathrm{h} \\
\text { (m2) }\end{array}$ & 4.61 & 4.32 & 4.99 & 5.96 & 6.20 & 5.216664 \\
\hline Relación & 1.18 & 1.17 & 1.16 & 1.14 & 1.13 & \\
\hline
\end{tabular}

Fuente. Elaboración propia.

Se aprecia que, con el paso del tiempo, se ha tendido a aumentar el espesor de la losa superior, así como de la inferior. Esto es debido a la importancia del concepto de losa de compresión. A la par que se ha aumentado la separación S entre almas, se recortado el largo de los voladizos, por lo que su espesor disminuye.

Además, debe mencionarse la tendencia de las teorías más novedosas a arrojar resultados de área transversal en $\mathrm{H}$ y h más altos que las teorías predecesoras, mientras que la relación entre ambas va disminuyendo. Una de las causas de esta evolución es el constante desarrollo de las normativas y acciones de diseño. Esto significa que, como se verá más adelante; se tiende a que la curvatura de la sección longitudinal no sea hoy en día tan pronunciada como lo era en los diseños antiguos.

\section{PROCESO CONSTRUCTIVO}

Los puentes en voladizos sucesivos se construyen por etapas [2] [4] [13] [14] [15] [16]. Cada etapa corresponde a la construcción de cada una de las dovelas que forman el puente, como se representa esquemáticamente en la figura 4. Este proceso de construcción se puede dividir en tres partes, según su orden de ejecución. Estas son Operaciones Preliminares (Dovela de pila), Operaciones Básicas (Ejecución de voladizos sucesivos) y Operaciones Posteriores (Dovela de cierre). 


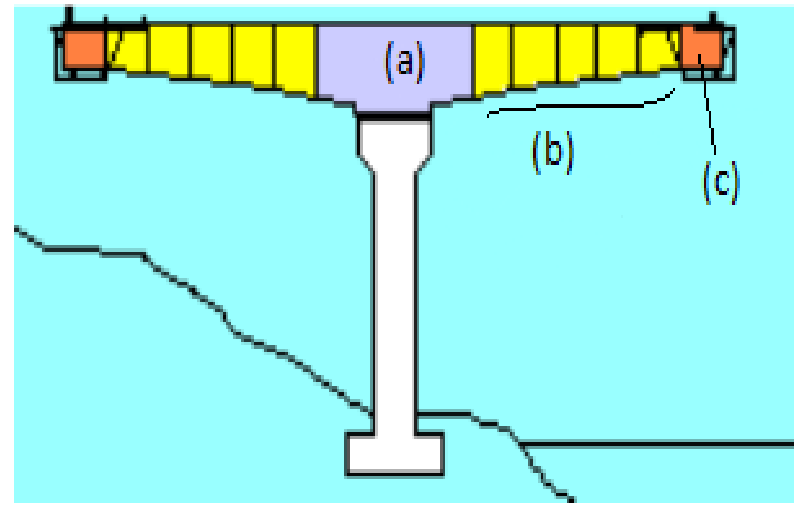

Figura 4. Vista de la sección longitudinal de un puente donde se aprecian (a) la dovela inicial, (b) las dovelas ya construidas y (c) las dovelas que se están construyendo. Fuente. SETRA.

La construcción por avance en voladizo consiste en la ejecución de las dovelas (entre 3 y 5 metros de longitud) a ambos lados de la pila, de forma simétrica. El vaciado se realiza con la ayuda de los encofrados, que a su vez se apoyan en las dovelas anteriores mediante cimbras para garantizar la continuidad del puente, por medio de cables de postensado. Vaciando las dovelas sucesivamente sobre los carros, se va avanzando desde las pilas hasta el centro del vano conectando mediante la dovela clave con el último voladizo del grupo perteneciente a la pila contigua.

El inicio de todo el proceso se da con la construcción de la dovela situada encima de la pila o también denominada dovela " 0 " o dovela de pila. Esta dovela se construye con un encofrado montado sobre la pila con longitud suficiente para que se puedan montar los carros de avance. Esto se aprecia en la figura 5.

\subsection{Operaciones Preliminares (Dovela de Pila)}

Se vacía la dovela de la pila o dovela "0". Inicialmente se construye la losa inferior de la sección, montando su formaleta, seguido del encofrado del alma y terminando con el acero de refuerzo y vaciado de la losa superior. Finalmente se retira toda la formaleta. Se puede concebir tanto quedando esta dovela empotrada en la pila, como simplemente apoyada sobre ella.

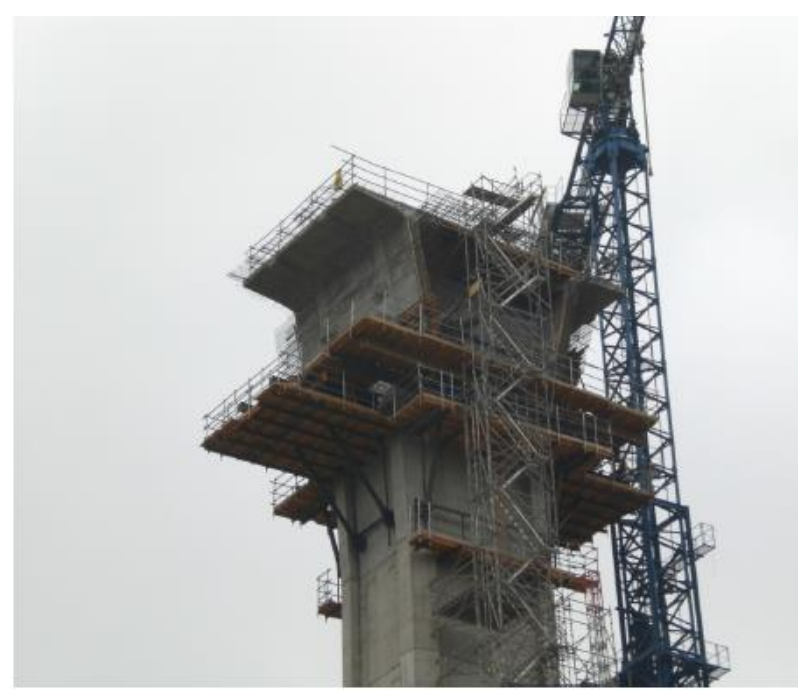

Figura 5. Vista de la ejecución de la dovela 0 en el viaducto de Tenoya, en Tenerife (España). Fuente. https://elblogingenierocivil.wordpress.com/

\subsection{Operaciones Básicas de Ciclo (Ejecución de voladizos sucesivos)}

\subsubsection{Carro de avance.}

El carro de avance es el medio más común que se tiene para la construcción del puente por voladizos sucesivos. Sirve para trasladar los encofrados y fundir las dovelas. Es la zona de trabajo de los operarios. Se muestra en las figuras 6,7 y 8 .

El carro está formado por las vigas metálicas principales, las vigas transversales, las vigas de rodadura, los apoyos frontales y traseros, los pisos de trabajo y los elementos y plataformas auxiliares.

Tras el montaje de la dovela de pila, se incorpora a ella el primer carro para la construcción de la dovela 1. Una vez finalizada el carro se desplaza para la construcción en voladizo de las dovelas sucesivas de dicho tramo. Así se libera la cabeza de pila para que pueda recibir al segundo carro que se ocupará de la construcción del tramo simétrico.

Una vez montado el carro de avance, se hacen revisiones periódicas tanto en el avance del carro, como en el vaciado; ya que en estos momentos son donde se dan las solicitaciones más críticas. 


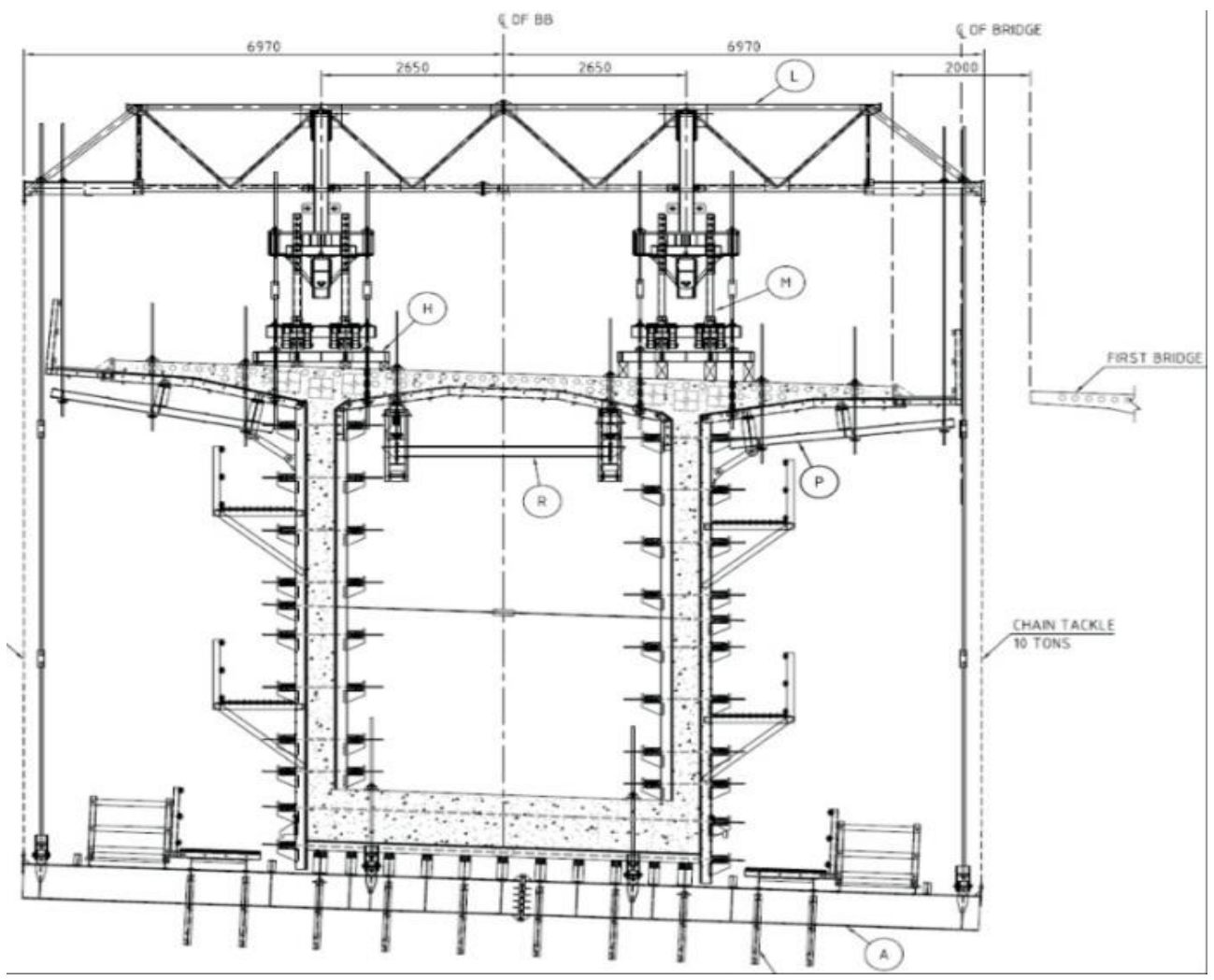

Figura 6. Vista en elevación del carro de avance utilizado en el puente Chilina (Perú). Fuente. http://www.puentechilina.com/carros-de-avance

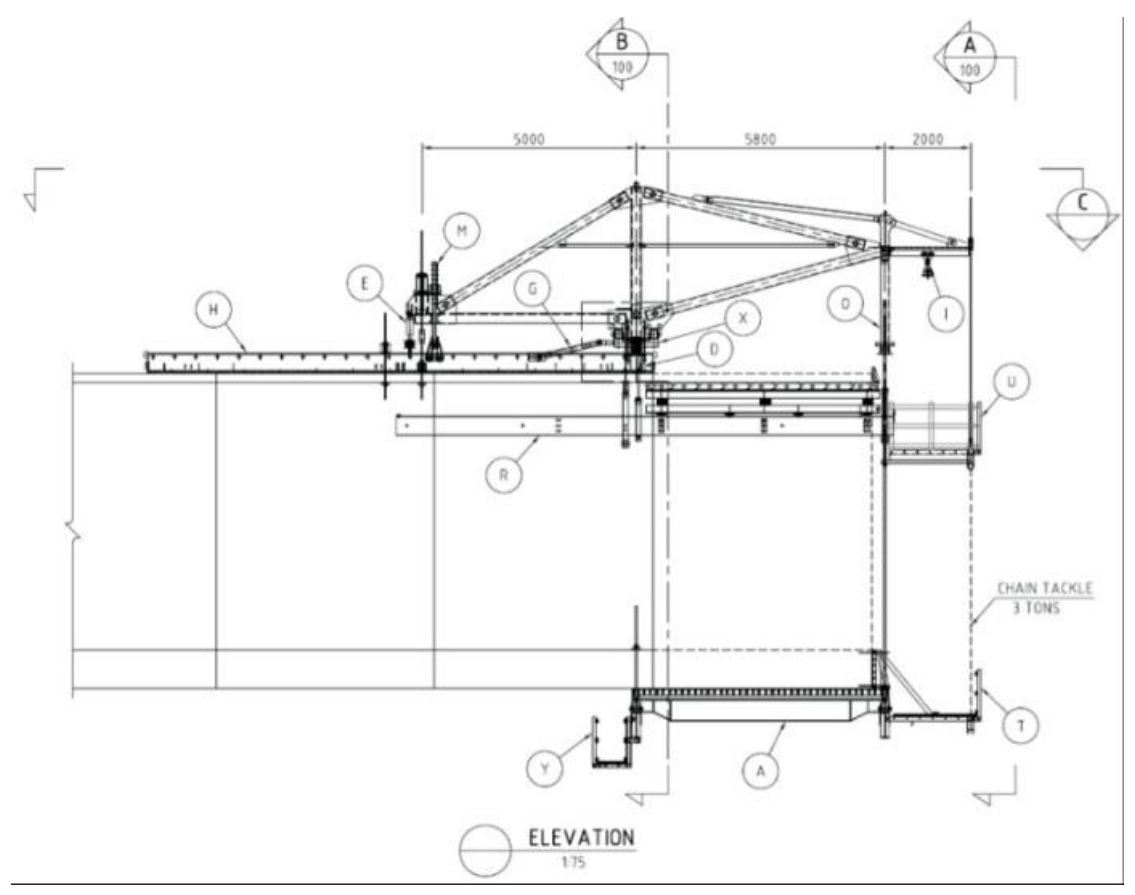

Figura 7. Vista en perfil del carro de avance utilizado en el puente Chilina (Perú). Fuente. http://www.puentechilina.com/carrosde-avance/ 


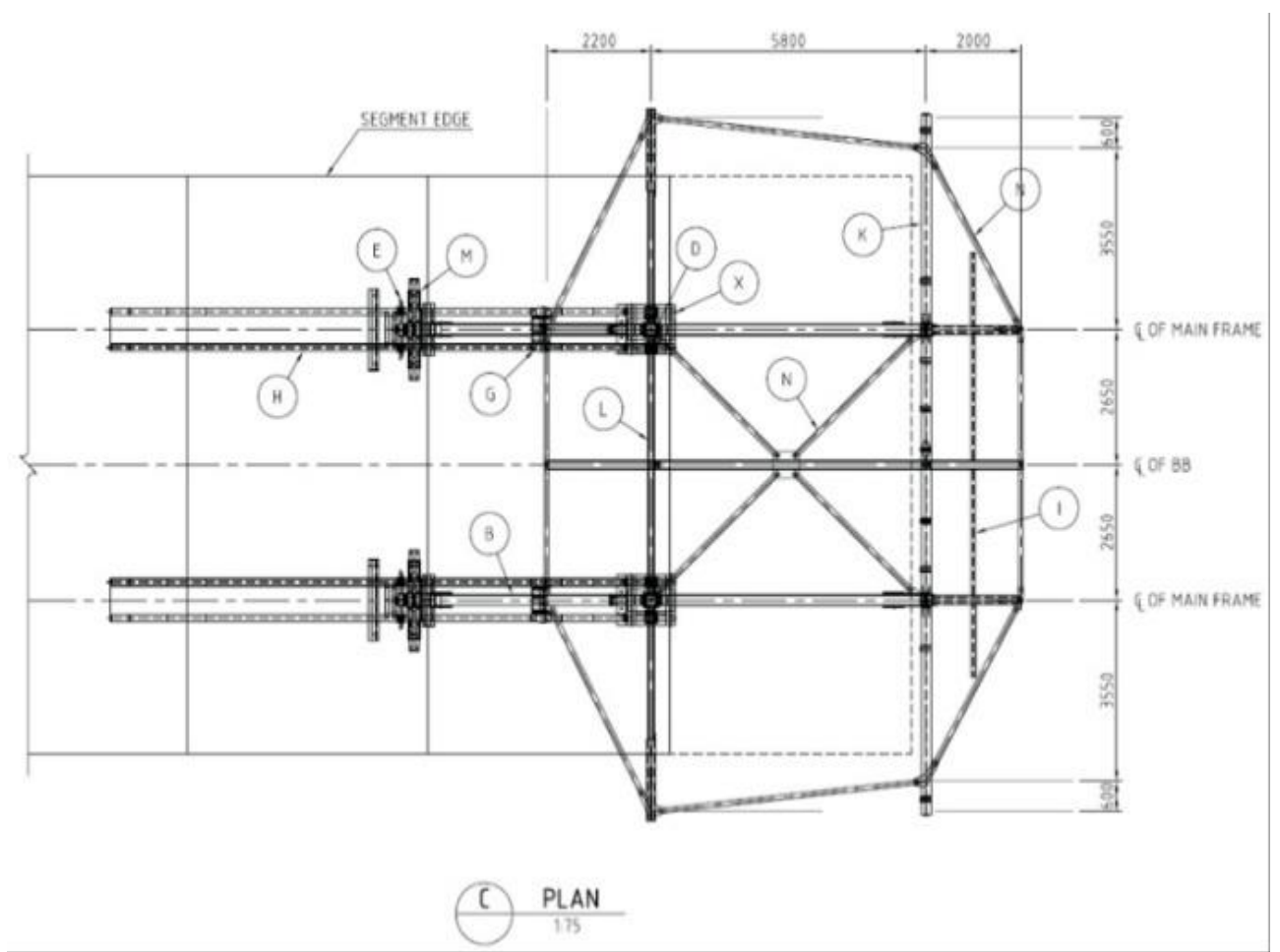

Figura 8. Vista en planta del carro de avance utilizado en el puente Chilina (Perú). Fuente. http://www.puentechilina.com/carrosde-avance/

\subsubsection{Ejecución de dovelas.}

A continuación, se describen las operaciones para ejecutar una dovela con vaciado in situ.

- Avance y fijación del carro. Es el primer paso. Comienza cuando el hormigón alcanza la resistencia necesaria para ser tensado y puede soportar el carro de avance.

- Colocación del acero de refuerzo inferior. Se monta manualmente el acero de refuerzo de la losa inferior.

- Formaleta. Se formaletea el resto de la sección.

- Colocación del acero de refuerzo superior. Se monta el acero de refuerzo de la losa superior y las vainas de los cables.

- Nivelación del carro. Paso previo al vaciado.

- Vaciado de la dovela. Durante el proceso, el carro se sujeta en los gatos y se ancla al tablero. A medida que se va reduciendo el canto, se deben desmontar los paneles sobrantes. Primero se hormigona la losa inferior y se va ascendiendo para terminar con la losa superior.

- Desmontaje de la formaleta de las dovelas.

- Curado del concreto. Debe evitarse la aparición de fisuras.

- Enfilado y tensado de los cables.

- Inyección de las vainas.

\subsection{Operaciones Posteriores (Dovela de cierre)}

Finalizado el vaciado de las dos últimas dovelas y antes de ejecutar la dovela de cierre, se procede a desmontar el carro de avance.
A continuación, se vacía la dovela de cierre, que es la encargada de unir los dos tramos que se han ido construyendo por voladizos sucesivos. Este momento es importante porque se transforman dos voladizos independientes entre sí en un vano que trabaja como una viga sustentada en dos apoyos. Para ejecutar la dovela de cierre, se inmovilizan, con vigas metálicas; los dos semivanos. Estos semivanos son independientes entre sí porque provienen de distintas pilas. Así se puede utilizar la plataforma inferior de uno de los carros como superficie de trabajo. Se utilizan formaletas distintas a las anteriores; ya que estas son mucho más ligeras.

Finalmente, se realiza el tensado de continuidad, produciéndose así la unión de los dos voladizos y convirtiéndolos en una viga continúa preparada para absorber las cargas móviles estipuladas. Dentro de la zona hueca del tablero se introducen los equipos de enhebrado de cables de postensado. Después éstos se enfilan para posteriormente tensarlos y darle continuidad a la estructura. Para introducir los cables se utilizan los ductos que se han dejado en la losa superior para dicho propósito. Las vainas de los cables de continuidad se inyectan también desde la losa superior del tablero.

Finalmente se deben tapar los agujeros que se dejan para el anclaje del carro de avance, así como la instalación de juntas de dilatación si están proyectadas sobre el tablero. 


\subsection{Variante de construcción por voladizos sucesivos}

Hasta ahora sólo se ha contemplado la construcción desde los pilares, pero hay una variante de esto. Cuando se debe salvar un vano pequeño, que no requiere pilares; se construye desde los estribos para unirse en el centro del vano como se muestra en la figura 9 .

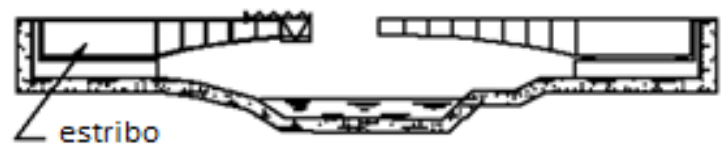

Figura 9. Construcción por voladizos sucesivos desde los estribos. Fuente. SETRA.

Para ello se colocan contrapesos en los estribos que soportan el peso de lo que se está construyendo hasta ahora como se muestra en la figura 10.

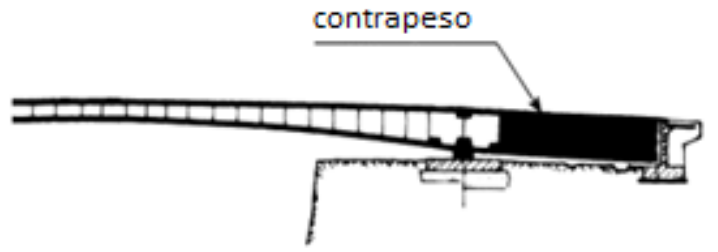

Figura 10. Vista de perfil de los contrapesos que sujetan los voladizos. Fuente. SETRA.

\subsection{Variación de momentos durante la construcción}

Una de las características de este tipo de construcción es que, durante su construcción, la estructura sufre diversas solicitaciones que pueden llegar a contrarrestarse con el paso del tiempo.

\subsubsection{Tensiones iniciales.}

La primera etapa abarca la construcción de los voladizos desde las pilas con el carro de avance mientras se van tensionando los cables

En este momento de la construcción sólo se dan momentos negativos como se muestra en la figura 11.
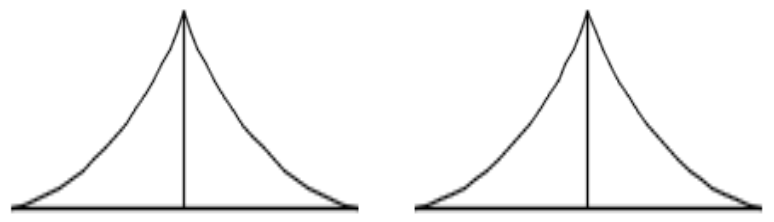

Figura 11. Momentos en la fase de construcción de los voladizos desde los pilares. Fuente. Elaboración propia.

\subsubsection{Tensiones al unirse al estribo.}

Cuando toman continuidad uniéndose al estribo aparecen los momentos positivos en la estructura como se muestra en la figura 12 .

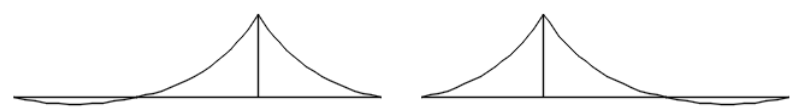

Figura 12. Momentos en la fase de unión a los estribos. Fuente. Elaboración propia.

\subsubsection{Tensiones finales.}

Al darle continuidad a la totalidad de la estructura se observa que el valor de los momentos negativos disminuye, los momentos positivos aumentan y además aparecen éstos en la unión de los voladizos como se muestra en la figura 12.

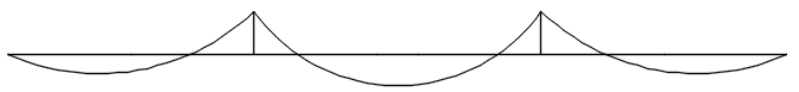

Figura 13. Momentos en la fase de unión a los voladizos sucesivos. Fuente. Elaboración propia.

\subsection{Evolución del proceso de diseño y constructivo}

Desde los primeros cálculos y construcciones de este tipo de puentes hasta hoy en día se han dado varios tipos de culminación de obra [3]. Se muestran a continuación por orden cronológico.

\subsubsection{Puentes de primera generación.}

Este periodo abarca los años entre 1953 y 1964. Los tableros de este tipo de puentes llamados de primera generación se construían unidos monolíticamente tanto a los pilares como a los estribos. Además, los voladizos entre ellos no se unían dándole continuidad, sino que se utilizaban las uniones machihembradas.

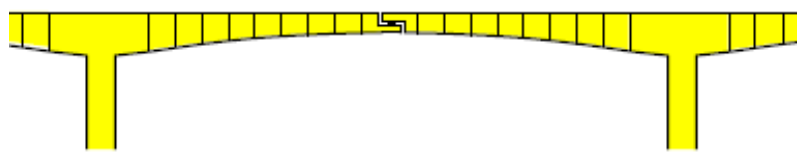

Figura 14. Detalle típico de la unión machihembrada de los puentes de primera generación. Fuente. SETRA.

En 1957 se construyó el puente Casey y el puente Beaucaire con esta unión machihembrada. Este tipo de puentes han tenido muchos problemas de deflexiones a lo largo de la historia; y por esta razón estos dos puentes fueron demolidos en 1973 y 1995 respectivamente. A 
raíz de estas experiencias han aparecido los denominados puentes de segunda generación.

\subsubsection{Puentes de segunda generación.}

Como los puentes de primera generación sufrieron una deformación excesiva de sus elementos en voladizo con el paso del tiempo, debido a una subestimación de los efectos de la fluencia lenta del hormigón y la contracción, se evolucionó a lo que se denomina segunda generación de puentes, que abarca desde el año 1965 al 1975. La diferencia fundamental entre los puentes de ésta generación y de la primera es que se contempla una estructura continua y monolítica; es decir, sin la unión machiembrada entre los voladizos en mitad del tramo. Se muestra la diferencia en las figuras 14 y 15 .

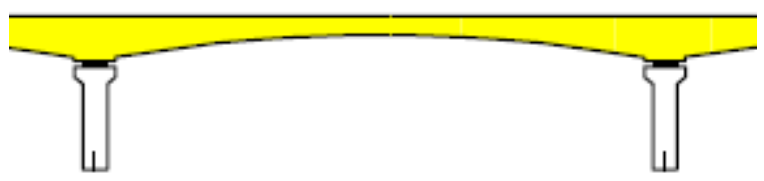

Figura 15. Detalle típico de la continuidad de la estructura de los puentes de segunda generación. Fuente. SETRA.

Este fue un período de enormes desarrollos y regulaciones que cambiaban constantemente en base a la experiencia del momento. También se aprecia como estos puentes de segunda generación aún tuvieron que repararse y repotenciarse con el paso de los años. Todos los progresos que se dieron allanaron el camino a la llamada tercera generación.

\subsubsection{Puentes de tercera generación.}

Se denomina así a los puentes diseñados y construidos entre 1975 y 1982. La diferencia con las generaciones anteriores es que estos puentes ya cuentan en el cálculo con la fluencia y el gradiente térmico, siendo estos factores muy importantes en la vida útil de la estructura.

Por tanto, se consideran estructuras mejor diseñadas que las anteriores y hoy en día no sufren los problemas que han sufrido los puentes de otras generaciones.

Se aprecia que ya en esta generación se tratan nuevas tecnologías de diseño como variar el tipo de hormigón o construir luces no balanceadas.

\subsection{4 Época actual.}

En esta época ya se generalizan los reglamentos de los cálculos estructurales; y además se introduce el concepto de control de calidad. Por otra parte, se desarrolla el pretensado exterior.

\section{SELECCIÓN DE PUENTES A ESTUDIAR}

Se tiene datos de 124 puentes [17] [18] [19] construidos en voladizos sucesivos in situ. Para considerar un puente como válido en este estudio se ha debido obtener mínimo el valor de la luz principal (L), el país al que pertenece y su fecha de diseño. Este grupo de datos se ha clasificado a su vez siguiendo tres criterios:

- Cronología

- Luz libre

- Países

A su vez, se ha estudiado según los criterios anteriores; la relación entre la luz principal y el canto en apoyos y el canto en el centro (L/H y L/h) así como la relación entre los dos cantos $(\mathrm{h} / \mathrm{H})$.

Además, dentro de esos tres criterios la se ha medido relación entre su luz principal (L) y las relaciones anteriores $(\mathrm{L} / \mathrm{H}, \mathrm{L} / \mathrm{h}$ y h/H). Estos resultados se comparan con los clásicos L/50 y L/20. Seguidamente se presentan gráficas paramétricas donde se aprecian los campos de aplicación de esta tipología.

\subsection{Cronología}

Se presentan los datos clasificados en orden cronológico. Esto es, se dividen los datos en tres grupos. Estos son:

- $1960-1980$

- 1980-2000

- 2000-2016

\subsubsection{0-1980.}

Se tienen datos geométricos de 50 puentes diseñados y construidos durante estos años, pertenecientes a 13 países (España, Colombia, Francia, Alemania, Noruega, Japón, Suiza, EE. UU, Liberia, Palau, Camerún, Serbia y Portugal). La luz principal (L) de estos puentes tienen valores que oscilan entre 44 metros y 241 metros.

\subsubsection{0-2000.}

Se tienen datos geométricos de 48 puentes diseñados y construidos durante estos años, pertenecientes a 16 países (España, Francia, Alemania, Noruega, Escocia, Bangladesh, Grecia, Suiza, EE. UU, Suecia, Finlandia, Tailandia, Polonia, Croacia, Reino Unido y Vietnam). La luz principal (L) de estos puentes tienen valores que oscilan entre 30 metros y 301 metros.

\subsubsection{0-2016.}

Se tienen datos geométricos de 27 puentes diseñados y construidos durante estos años, pertenecientes a 13 países (España, Colombia, Francia, Alemania, Noruega, Bolivia, Turquía, Grecia, Malasia, República Checa, Abu 
Dhabi y Australia). La luz principal (L) de estos puentes tienen valores que oscilan entre 44 metros y 298 metros.

\subsection{Luz libre}

Se presentan los datos clasificados por magnitud de luz libre. Se tiene valores de hasta 301 metros y se presentan fraccionados en grupos de cada 50 metros. Estos son:

- $<50$

- 50-100

- $100-150$

- $150-200$

- $200-250$

- 250-301

4.2.1 $<50 \mathrm{~m}$.

Se tienen datos geométricos de 4 puentes con una luz libre inferior a 50 metros, pertenecientes a 3 países (Francia, Alemania, Tailandia). Estos puentes fueron construidos entre los años 1977 y 2004.

\subsubsection{0m-100m.}

Se tienen datos geométricos de 44 puentes con una luz libre de entre 50 metros y 100 metros, pertenecientes a 9 países (España, Francia, Colombia, Alemania, Suiza, Malasia, Polonia, Croacia, Portugal). Estos puentes fueron construidos entre los años 1957 y 2007.

\subsubsection{0m-150m.}

Se tienen datos geométricos de 46 puentes con una luz libre de entre 100 metros y 150 metros, pertenecientes a 16 países (España, Francia, Colombia, Alemania, Noruega, Bolivia, Bangladesh, Suiza, EE. UU, Suecia, Liberia, Finlandia, Tailandia, República Checa, Camerún, Vietnam). Estos puentes fueron construidos entre los años 1967 y 2011.

\subsubsection{0m-200m.}

Se tienen datos geométricos de 17 puentes con una luz libre de entre 150 metros y 200 metros, pertenecientes a 8 países (España, Francia, Colombia, Alemania, Noruega, Suiza, Abu Dhabi, Turquía). Estos puentes fueron construidos entre los años 1968 y 2014.

\subsubsection{0m-250m.}

Se tienen datos geométricos de 11 puentes con una luz libre de entre 200 metros y 250 metros, pertenecientes a 9 países (Francia, Alemania, Escocia, Japón, Grecia, EE. UU, Palaus, Serbia, Reino Unido). Estos puentes fueron construidos entre los años 1960 y 2004.

\subsubsection{0m-301m.}

Se tienen datos geométricos de 3 puentes con una luz libre de entre 250 metros y 301 metros, siendo esta la luz mayor de las estudiadas; pertenecientes a 2 países
(Noruega y Australia). Estos puentes fueron construidos entre los años 1998 y 2007.

\subsection{Países}

Se presentan los datos clasificados por el país donde se haya construido. Se forman cuatro grupos pertenecientes cada uno a una nación distinta, debido al volumen considerado de datos de ese país; y un quinto grupo donde se engloban el resto de puentes del resto del mundo. Estos son:

- Alemania

- Colombia

- Francia

- España

- Resto del mundo

\subsubsection{Alemania.}

Se tienen datos geométricos de 12 puentes en Alemania, con una luz libre (L) entre 40 metros a 208 metros. Pertenecen al periodo temporal de los años 1960-2004.

\subsubsection{Colombia.}

Se tienen datos geométricos de 7 puentes en Colombia, con una luz libre (L) entre 62 metros a 200 metros. Pertenecen al periodo temporal de los años 1975-2007.

\subsubsection{Francia.}

Se tienen datos geométricos de 53 puentes en Francia, con una luz libre (L) entre 30 metros a 242 metros. Pertenecen al periodo temporal de los años 1960-2011.

\subsubsection{España.}

Se tienen datos geométricos de 15 puentes en España, con una luz libre (L) entre 80 metros a 190 metros. Pertenecen al periodo temporal de los años 1972-2014.

\subsubsection{Resto del mundo.}

Se tienen datos geométricos de 38 puentes en Noruega, Escocia, Japón, Bolivia, Bangladesh, Turquía, Grecia, Suiza, EE. UU, Suecia, Liberia, Malasia, Finlandia, Palau, Tailandia, Polonia, Croacia, República checa, serbia, Portugal, Camerún, Portugal, Abu Dhabi, Australia, Reino Unido y Vietnam. Pertenecen al periodo temporal de los años 1969-2008.

\section{4 Árbol lógico}

Se presenta en la figura 16 un árbol lógico donde se sintetiza la forma en la que se han discretizado los datos tratados. 


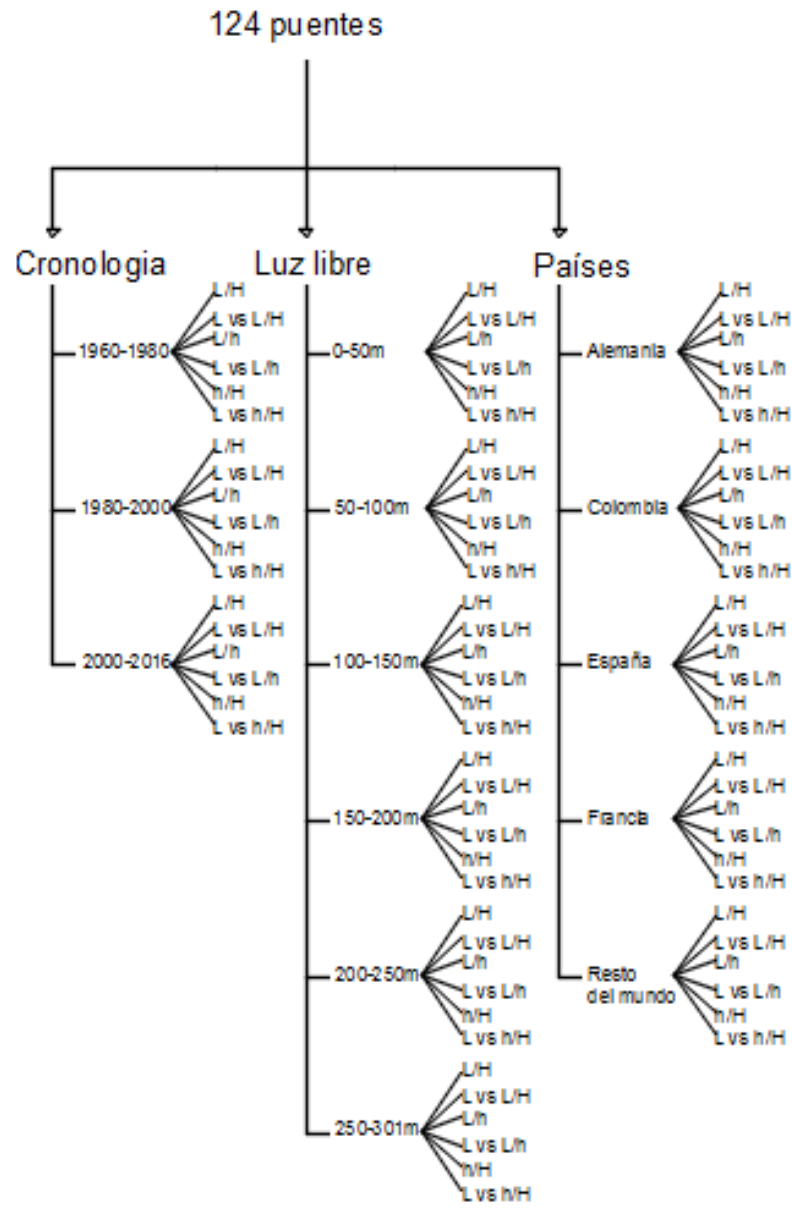

Figura 16. Árbol lógico referente a los datos de estudio. Fuente. Elaboración propia.

\section{ANÁLISIS ESTADÍSTICO}

Se realiza un análisis estadístico de los datos pertenecientes a los puentes presentados. Las operaciones a las que han sido sometidos los grupos de datos son las siguientes, mostradas a continuación en orden cronológico:

- Desviación estándar $(\sigma)$

- Descarte de los de fuera de rango

- Regresión lineal y límites resultantes de aplicación

- Comparativa con los conceptos clásicos de L/20 y L/50

A continuación, a modo de ejemplo ilustrativo de lo anterior; se muestra este proceso para el grupo $\mathrm{L} / \mathrm{H}$, de los años 1960-1980 dentro de la discretización cronológica de los datos.

\subsection{Desviación estándar}

En el grupo de datos de L/H de los años 1960-1980 de la discretización cronológica de los datos se aplica el concepto de desviación estándar para eliminar los que se consideren fuera de rango (5\%) para que los datos restantes se consideren sólidos y fiables, es decir; con una correlación cercana a 1 .

Se muestra en la figura 17 el gráfico de barras de este grupo, siendo cada barra un puente perteneciente al grupo estudiado y reflejándose en el eje vertical el valor de la relación $\mathrm{L} / \mathrm{H}$ respectivo. También se muestra la desviación estándar $(\sigma)$ para apreciar como los puentes que queden fuera de rango no se estudiarán.

En este caso la media del valor $\mathrm{L} / \mathrm{H}$ es de 18.58 , el valor de la desviación estándar $\sigma$ resulta de 3.32 , por lo que quedan fuera de estudio los puentes cuyo valor $\mathrm{L} / \mathrm{H}$ sea menor a 15.25 y mayor a 21.90 .

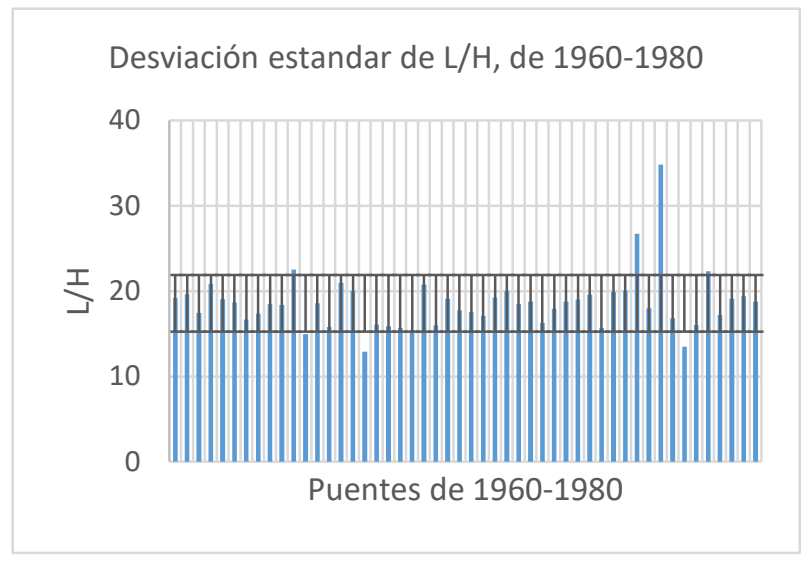

Figura 17. Gráfico de barras donde se representa el cálculo de la desviación estándar para el grupo mencionado. Fuente. Elaboración propia.

\subsection{Descarte de los de fuera de rango}

Continuando con el ejemplo de cómo se ha estudiado este grupo de datos, se eliminan los puentes que la desviación estándar $(\sigma)$ ha dejado fuera de rango. En este caso, de los 50 puentes se van a estudiar 43 y se dejan fuera de estudio 7.

\subsection{Regresión lineal y límites resultantes de aplicación}

Una vez eliminados los datos de los puentes desechados por la desviación estándar $\sigma$ se grafica la relación que se está estudiando. Continuando con este ejemplo ilustrativo, se grafica en la figura 18 de forma paramétrica $\mathrm{L} / \mathrm{H}$ de los 43 puentes considerados.

Además de eso se establece una recta de regresión lineal de los datos. Con el valor de la desviación estándar $\sigma$ se fijan límites superior e inferior, quedando representado el rango de aplicación de este grupo de datos. 
Es importante remarcar que la correlación es de 0.94. Por esa razón se consideran estos datos utilizados como sólidos y fiables.

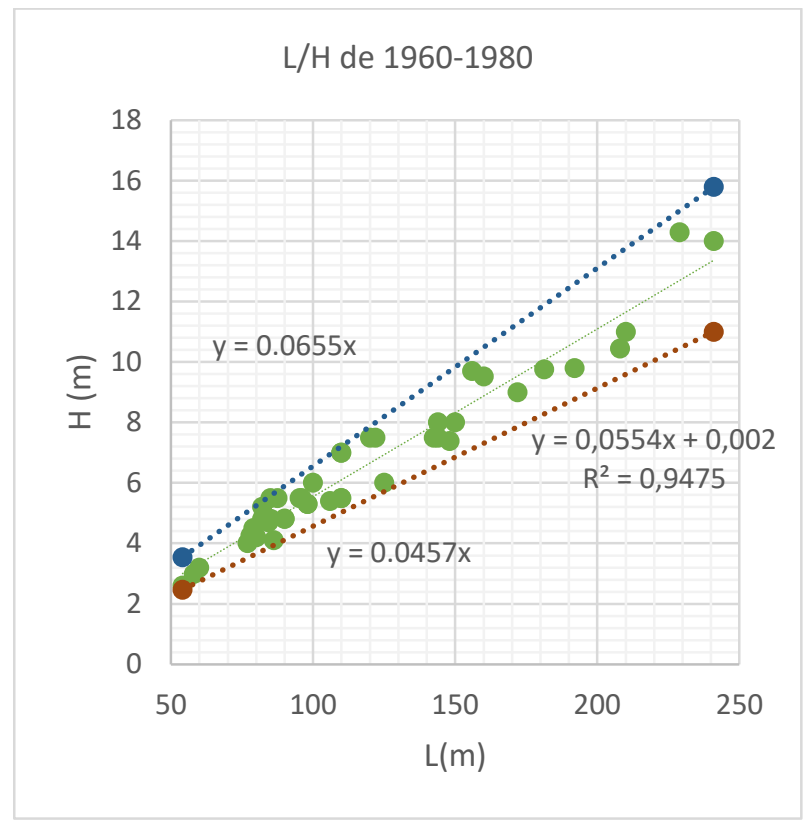

Figura 18. Gráfico paramétrico de dispersión donde se muestra para este grupo de estudio (a) el valor de $\mathrm{L} / \mathrm{H}$ de cada puente, (b) los límites superior e inferior del rango estudiado aplicando la desviación estándar $\sigma$ mencionada y la ecuación que los describe, (c) la línea de regresión lineal y la ecuación que la describe y (d) la correlación entre los datos estudiados. Fuente. Elaboración propia.

\subsection{Comparativa con los conceptos clásicos de $L / 20$ y L/50}

Se ha hallado la relación entre el valor estudiado y el valor L del puente. En este caso de ejemplo se estudia la relación entre $\mathrm{L} / \mathrm{H}$ y $\mathrm{L}$. Como $\mathrm{H}$ corresponde al valor del canto del tablero en los apoyos, se procede a compararlo con el correspondiente $\mathrm{L} / 20$.

En este caso se observa en la figura 19 que los puentes estudiados varían su relación $\mathrm{L}$ vs $\mathrm{L} / \mathrm{H}$ entre los valores de $L / 15.25$ y L/21.90.

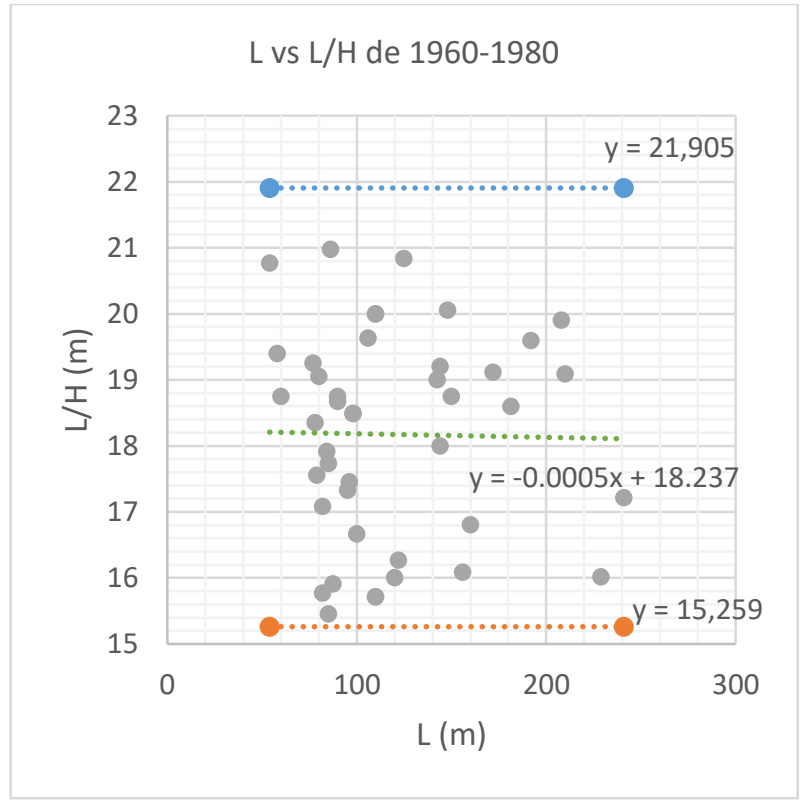

Figura 19. Gráfico de dispersión donde se muestra para este grupo de estudio (a) el valor de $\mathrm{L}$ vs $\mathrm{L} / \mathrm{H}$ de cada puente, (b) los límites superior e inferior del rango estudiado aplicando la desviación estándar $\sigma$ mencionada y la ecuación que los describe, y (c) la línea de regresión lineal y la ecuación que la describe. Fuente. Elaboración propia.

\section{ANÁLISIS DE RESULTADOS}

Se presenta a continuación el análisis de resultados del estudio estadístico anterior. Se muestra según los tres apartados ya comentados; Cronología, Luz libre y Países.

\subsection{Cronología}

Según la figura 20, los valores extremos de la relación L vs $\mathrm{L} / \mathrm{H}$ con el paso del tiempo se van alejando del clásico $\mathrm{L} / 20$. El rango de amplitud variable de $\mathrm{H}$ en relación a $\mathrm{L}$ en 1980-2000 aumentó por su límite superior, manteniendo el inferior en el mismo valor, y en la actualidad (2000-2016) el límite superior ha descendido, pero el inferior también, como se demuestra en la gráfica siguiente. 


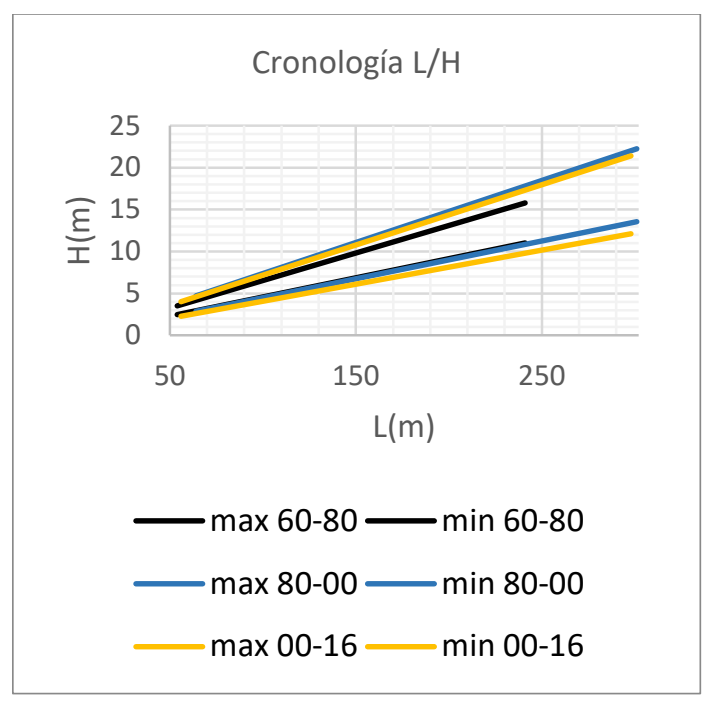

Figura 20. Gráfico de la variabilidad del rango de $\mathrm{H}$ frente a $\mathrm{L}$, donde se muestran los límite superior e inferior de este valor en (a) los años 1960-1980, (b) los años 1980-2000, y (c) los años 2000-2016. Fuente. Elaboración propia.

Los valores extremos de la relación $\mathrm{L}$ vs $\mathrm{L} / \mathrm{h}$, como se aprecia en la figura 21; con el paso del tiempo se van alejando del clásico L/50. El rango de amplitud variable de $\mathrm{h}$ en relación a L en 1980-2000 aumentó tanto por su límite superior como por el inferior, y en la actualidad (2000-2016) el rango de amplitud de h ha crecido. Esto es que ha aumentado su límite superior y ha disminuido su límite inferior, como se demuestra en la siguiente gráfica. Es importante mencionar que hoy en día se manejan valores del rango L/68-L/30, muy lejos del clásico L/50.

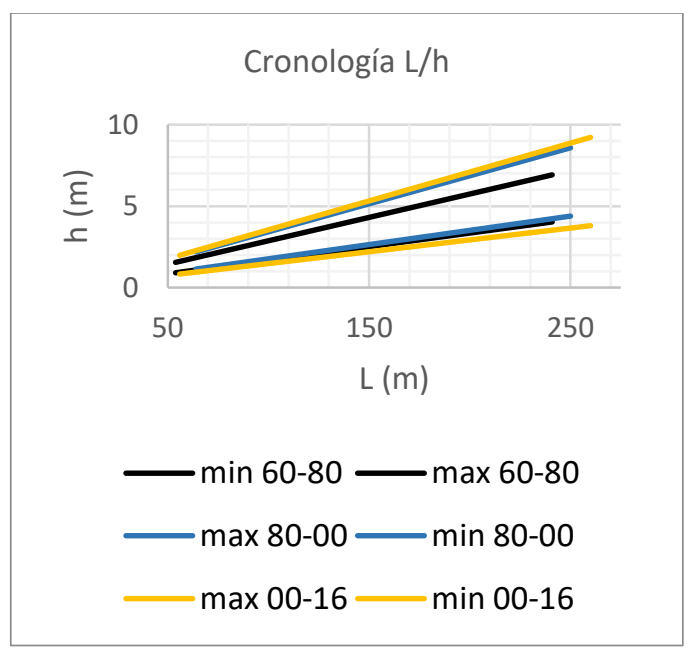

Figura 21. Gráfico de la variabilidad del rango de h frente a L, donde se muestran los límite superior e inferior de este valor en (a) los años 1960-1980, (b) los años 1980-2000, y (c) los años 2000-2016. Fuente. Elaboración propia.
Según se aprecia en la figura 22, los valores extremos superiores de la relación $\mathrm{L}$ vs $\mathrm{h} / \mathrm{H}$ con el paso del tiempo han ido variando, pero no así los inferiores, que se consideran similares en las tres épocas estudiadas. En la época de los años 1980-2000 el valor superior se redujo, haciendo los valores de h y H más similares entre sí; pero finalmente hoy en día puede considerarse (como se aprecia en la figura 21) que se ha vuelto a los valores de la primera época estudiada (1960-1980).

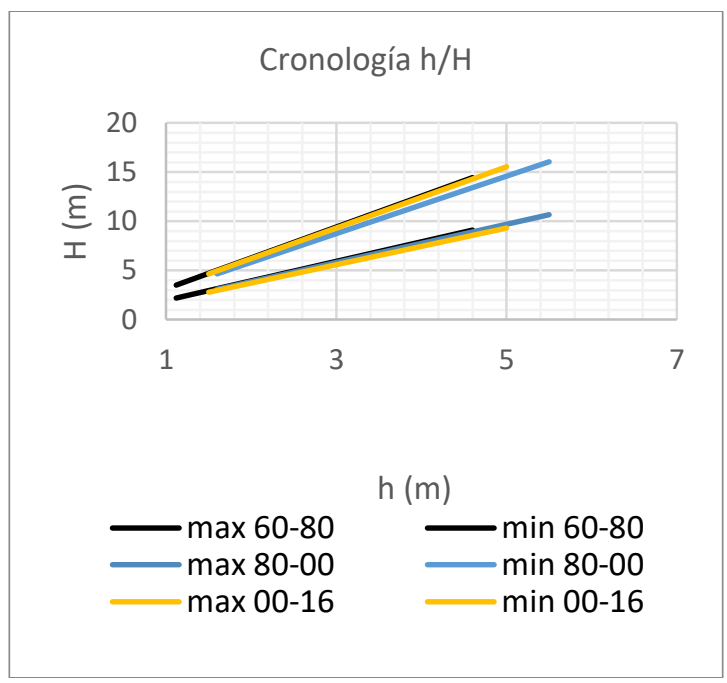

Figura 22. Gráfico de la variabilidad del rango de $\mathrm{H}$ frente a h, donde se muestran los límite superior e inferior de este valor en (a) los años 1960-1980, (b) los años 1980-2000, y (c) los años 2000-2016. Fuente. Elaboración propia.

A continuación, se exponen la figura 23, la figura 24 y la figura 25, donde se muestran las gráficas de la tendencia de variación del rango de las relaciones $\mathrm{L} / \mathrm{H}, \mathrm{L} / \mathrm{h}$ y h/H respectivamente, a lo largo de las épocas estudiadas.

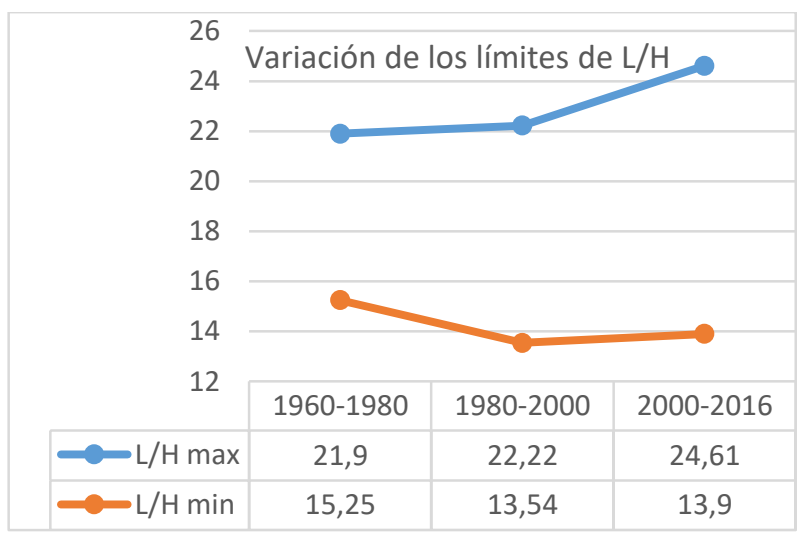

Figura 23. Gráfico de la evolución del rango de L/H en las épocas estudiadas. Fuente. Elaboración propia. 


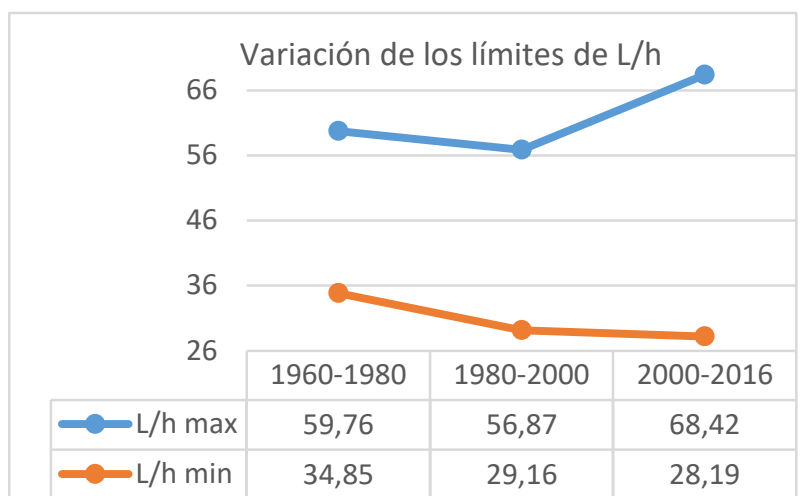

Figura 24. Gráfico de la evolución del rango de L/h en las épocas estudiadas. Fuente. Elaboración propia.

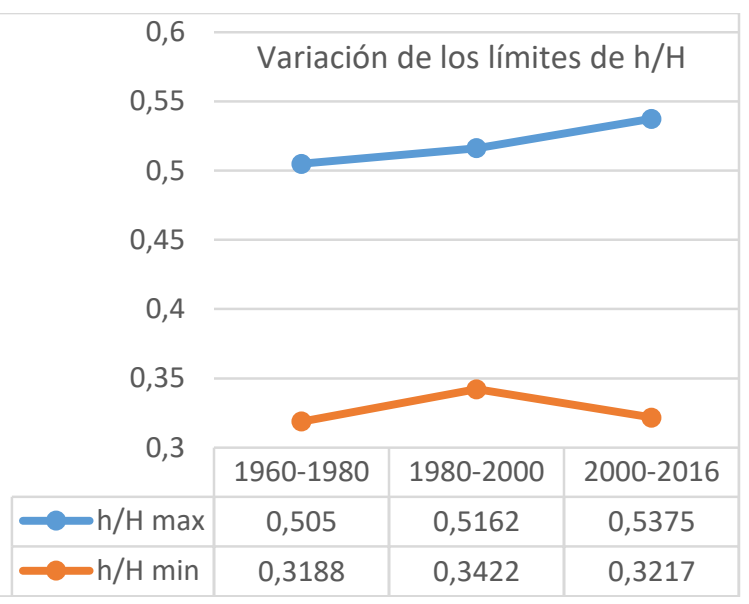

Figura 25. Gráfico de la evolución del rango de h/H en las épocas estudiadas. Fuente. Elaboración propia.

Puede concluirse que los rangos se van ampliando conforme avanza la historia, ya que también ha ido cambiando la forma de concebir este tipo de estructuras.

\subsection{Luz libre}

Se han divido los datos en grupos cada 50 metros de luz libre para valores inferiores a $301 \mathrm{~m}$.

Debe remarcarse que los resultados del estudio arrojan que este tipo de puentes no son apropiados para luces menores de $50 \mathrm{~m}$, así como para luces mayores de $250 \mathrm{~m}$, debido a que resultan relaciones $\mathrm{L} / \mathrm{H}, \mathrm{L} / \mathrm{h}$ y $\mathrm{h} / \mathrm{H}$ fuera de los rangos normales.

A continuación, se muestran la figura 26, la figura 27 y la figura 28, donde se exponen las gráficas de las relaciones estudiadas para cada grupo de datos.

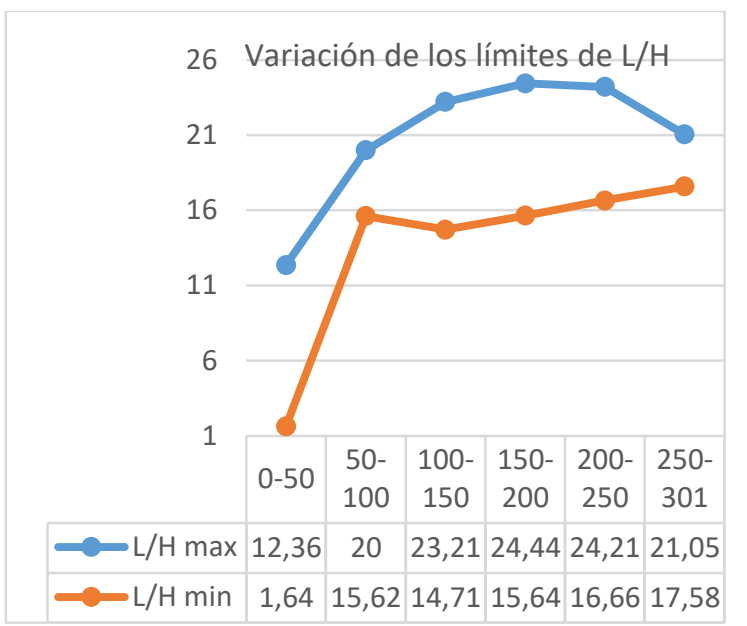

Figura 26. Gráfico de los rangos típicos de $\mathrm{L} / \mathrm{H}$ para cada valor de L estudiado. Fuente. Elaboración propia.

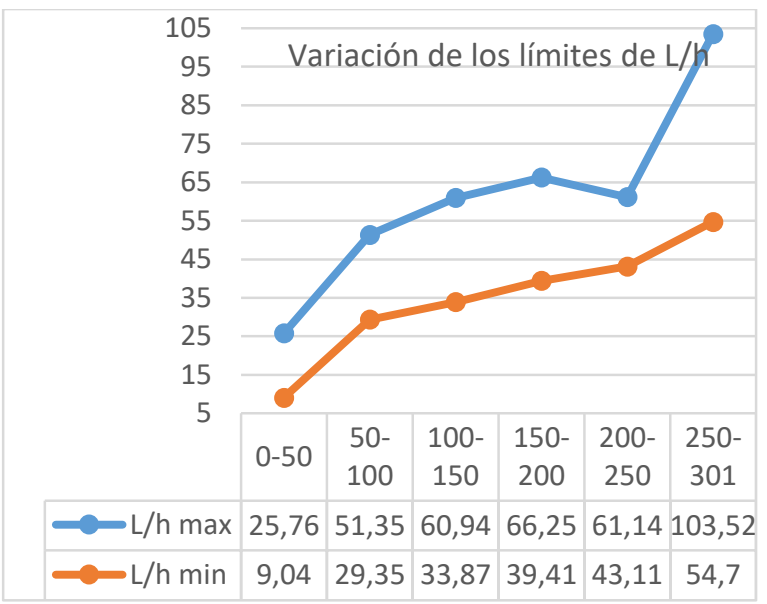

Figura 27. Gráfico de los rangos típicos de L/h para cada valor de L estudiado. Fuente. Elaboración propia.

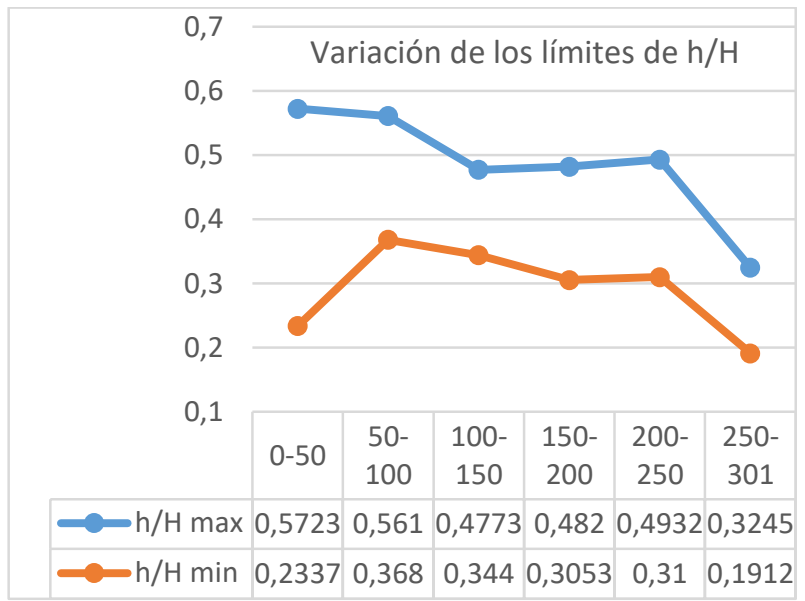

Figura 28. Gráfico de los rangos típicos de h/H para cada valor de L estudiado. Fuente. Elaboración propia. 
Como se mencionó, queda demostrado que para este tipo de puentes el rango aconsejable de luces es de entre $50 \mathrm{~m}$ y $250 \mathrm{~m}$. Para valores de luz libre fuera de ese rango, no resultan eficientes, como muestran las relaciones estudiadas en los tramos inferiores a $50 \mathrm{~m}$ y de $250 \mathrm{~m}$ a $301 \mathrm{~m}$.

Son ineficientes a luces menores de 50 metros porque en tan poca luz libre debe generar la curvatura estudiada, y eso lleva a valores no óptimos de $\mathrm{H}$ y h. Por otro lado, debido a la gran longitud de luces mayores a 250 metros, la relación $\mathrm{h} / \mathrm{H}$ resulta entre $1 / 3$ y $1 / 4$, mientras que en valores de $\mathrm{L}$ normales esta relación arroja resultados entre $1 / 3$ y $1 / 2$.

Respecto a las relaciones $\mathrm{L} / \mathrm{H}$ y $\mathrm{L} / \mathrm{h}$ se observa como en el rango descrito como típico de L se mantienen cerca de los clásicos L/20 y L/50, mientras que fuera de ese rango de $\mathrm{L}$ se distorsionan totalmente, dando lugar por ejemplo a L/1.64 para L/H o L/103.52 para L/h.

\subsection{Países}

Se han divido los 124 puentes según los países más representativos entre los que se encuentran. Se exponen los valores de las relaciones estudiadas.

En la figura 29 se presenta la gráfica de los valores por países de $\mathrm{L} / \mathrm{H}$, donde se ve que Colombia tiene el menor valor de $\mathrm{H}$ respecto a $\mathrm{L}$, es decir; contempla valores de $\mathrm{H}$ muy bajos. En el caso opuesto se encuentra Alemania, con valores de $\mathrm{H}$ altos en relación a $\mathrm{L}$.

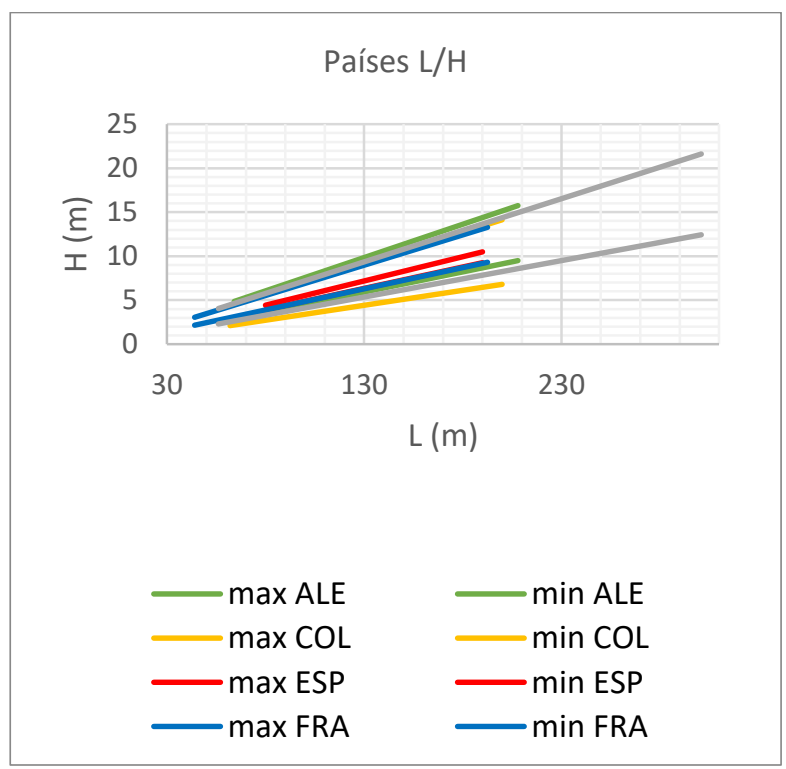

Figura 29. Gráfico de la variabilidad del rango de $\mathrm{H}$ frente a $\mathrm{L}$, donde se muestran el límite superior e inferior de este valor en (a) Alemania, (b) Colombia, (c) España, (d) Francia y (e) resto del mundo. Fuente. Elaboración propia.
En la figura 30 se presenta la gráfica de los valores por países de $\mathrm{L} / \mathrm{h}$, donde se ve que Colombia tiene el menor valor de $\mathrm{h}$ respecto a $\mathrm{L}$, es decir; contempla valores de $\mathrm{h}$ muy bajos. En el caso opuesto se encuentra Alemania, con valores de $\mathrm{h}$ altos en relación a $\mathrm{L}$.

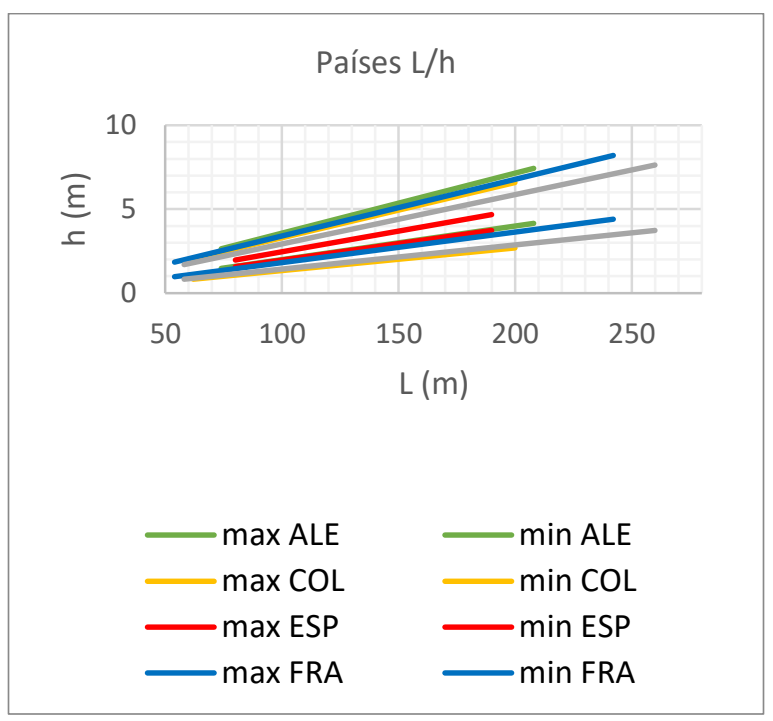

Figura 30. Gráfico de la variabilidad del rango de h frente a L, donde se muestran el límite superior e inferior de este valor en (a) Alemania, (b) Colombia, (c) España, (d) Francia y (e) resto del mundo. Fuente. Elaboración propia.

Se obtiene que Colombia tiene puentes muy esbeltos, (valores de $\mathrm{H}$ y h bajos) frente a Alemania, que tiene los valores más altos de $\mathrm{H}$ y h frente a la luz de los países estudiados.

En la figura 31 se presenta la gráfica de los valores por países de h/H, donde se ve que Colombia y España tienen un rango de valores de $\mathrm{h} / \mathrm{H}$ pequeño; es decir, no hay mucha variación entre los valores de $\mathrm{h}$ y $\mathrm{H}$ de sus puentes. El rango más amplio por otro lado, lo tiene Francia.

\section{CONCLUSIONES}

Se estudiaron 124 puentes de voladizos sucesivos in situ, construidos en 29 países. Se estudiaron las diferentes metodologías para el predimensionamiento. Se realizó un análisis estadístico y se obtuvieron gráficas paramétricas de las que se obtuvieron las siguientes conclusiones.

Del estudio de las diferentes teorías de predimensionamiento a lo largo de distintas épocas, se concluye que se tiende a aumentar la distancia entre almas y a aumentar el espesor de la losa superior e inferior. Además, se tiende a que la diferencia de áreas de la sección transversal asociada a los puntos donde se define $\mathrm{H}$ y h sea menor. Considerando, como es lo más 
habitual; que no varían los espesores a lo largo de la sección transversal, se obtiene que lo que varía es la altura de la viga. Esto lleva a concluir que se tiende a disminuir diferencia de altura de viga entre las secciones $\mathrm{H}$ y h, es decir; a una curvatura menor de la sección longitudinal.

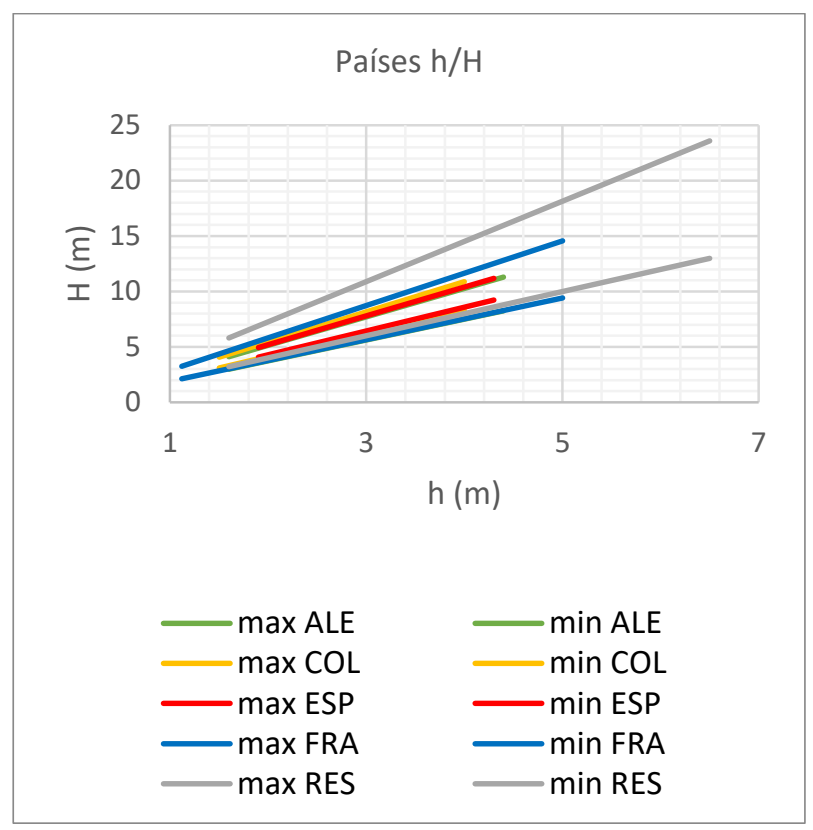

Figura 31. Gráfico de la variabilidad del rango de h frente a $\mathrm{H}$, donde se muestran los límites superiores e inferiores de este valor en (a) Alemania, (b) Colombia, (c) España, (d) Francia y (e) resto del mundo. Fuente. Elaboración propia

En el análisis cronológico se concluye que los valores extremos de la relación $\mathrm{L}$ vs $\mathrm{L} / \mathrm{H}$ con el paso del tiempo se van alejando del clásico L/20. Hoy en día el valor de $\mathrm{H}$ frente a $\mathrm{L}$ puede variar en un rango más amplio que en la época de las primeras estructuras estudiadas.

Igualmente, los valores extremos de la relación $\mathrm{L}$ vs $\mathrm{L} / \mathrm{h}$ con el paso del tiempo se van alejando del clásico L/50. En la actualidad el rango de amplitud de h ha crecido. Se debe mencionar que hoy en día se manejan valores del rango L/68-L/30, muy lejos del clásico L/50.

Del estudio por tramos se concluye que este tipo de puentes no son adecuados para luces menores de 50 metros, ni para mayores de 250 , ya que resultan valores de las relaciones $\mathrm{L} / \mathrm{H} \mathrm{y} \mathrm{L} / \mathrm{h}$ anormales y lejos de lo óptimo. En los puentes de luz menor de 50 metros la curva debe generarse en esa longitud, que resulta insuficiente. En los puentes de luz mayor a 250 metros debido a su gran longitud, la relación $\mathrm{h} / \mathrm{H}$ resulta entre $1 / 3$ y $1 / 4$, mientras que, en valores de $L$ normales, esta relación arroja resultados entre 1/3 y 1/2.
Respecto a las relaciones $\mathrm{L} / \mathrm{H}$ y $\mathrm{L} / \mathrm{h}$ se observa como en el rango anteriormente descrito de valores de $\mathrm{L}$ entre $50 \mathrm{~m}-250 \mathrm{~m}$ se mantienen cerca de los clásicos L/20 y $\mathrm{L} / 50$, mientras que fuera de ese rango de $\mathrm{L}$ se distorsionan totalmente, dando lugar por ejemplo a $\mathrm{L} / 1.64$ para $\mathrm{L} / \mathrm{H}$ o L/103.52 para $\mathrm{L} / \mathrm{h}$. Por eso este rango de luz libre se considera como óptimo para este tipo de puentes.

Del estudio por países se concluye que Colombia contempla valores de $\mathrm{H}$ y h muy bajos, en contraposición a Alemania, que es el país con valores $\mathrm{H}$ y h más altos. Además, se observa que Colombia y España tienen el rango de $\mathrm{h} / \mathrm{H}$ más pequeño, es decir; su $\mathrm{H}$ y h no varía mucho. Esta similitud se debe en parte a las obras ejecutadas en Colombia por empresas españolas. En el lado opuesto está Francia. Esta diferencia se da porque históricamente Francia ha sido muy continuista con su propia cultura ingenieril, sin tener en cuenta influencias externas.

\section{AGRADECIMIENTOS}

Se agradece el apoyo logístico y económico de Estructuras \& Sísmica S.A.S al desarrollo de esta investigación.

\section{REFERENCIAS}

[1] Obras de paso de nueva construcción, Ministerio de Fomento de España, Madrid, España, 2000

[2] D. F. Cetina, N.E. Ovalle, "Evaluación del proceso constructivo en el comportamiento estructural de un puente construido por voladizos sucesivos," Tesis de Grado, Facultad de Ingenierías Físico-Mecánicas, Universidad Industrial de Santander, Bucaramanga, 2011.

[3] Prestressed concrete bridges built using the cantiliver method-SETRA, Ministere de 1'Ecologie du Developpment et de 1'Amenagement durables, Francia, 2003

[4] W Podolny y J.M. Muller "Prestressed concrete bridges and segmental construction" en Construction and desing of prestressed concrete segmental bridges, 1a ed. Canada:Wiley-Interscience, 1982.

[5] W Podolny y J.M. Muller “Cast-in-place balanced cantiliver girder bridges" en Construction and desing of prestressed concrete segmental bridges, 1a ed. Canada:Wiley-Interscience, 1982. 
[6] C. F. Casado, "Ejecición de puentes pretensados por voladizos sucesivos," Informes de la construcción, vol. 16, no. 158, Mar. 1964

[7] J. Mathivat "Construcción en voladizo por hormigonado in-situ de dovelas" en Construcción de puentes de hormigón pretensado por voladizos sucesivos, 1a ed. Barcelona, España:Editores técnicos asociados, 1980.

[8] Precast segmental box girder bridge manual, PCI, EE.UU, 1978

[9] J. Mathivat "Concepción y dimensionamiento de tableros" en Construcción de puentes de hormigón pretensado por voladizos sucesivos, 1a ed. Barcelona, España:Editores técnicos asociados, 1980.

[10] J. Manterola “Construción de puentes” en Puentes. Apuntes para su diseño y construcción, 1a ed. Madrid, España:Colección Escuelas, 2006.

[11] F. A. Ariñez, "Criterios para la optimización del predimensionamieto de puentes de sección cajón," Tesis de Máster, Escuela Técnica Superior de Ingenieros de Caminos, Canales y Puertos, Universidad Politécnica de Madrid, Madrid, 2012.

[12] M. Aguiló, J. Manterola, M. Onzain.,J Rui-Wamba Javier Manterola Armisén. Pensamiento y Obra, 1a ed. Madrid, Fundación ESTEYCO, 2004.

[13] C. Luna, “Análisis y dimensionamietno de puentes construidos por avance de cimbra," Trabajo Fin de Grado, Escuela Técnica Superior de Ingenieros de Sevilla, Universidad de Sevilla, Sevilla, 2015.

[14] M. L. Nancu, "Metodología para el Análisis y Dimensionamiento de Puentes Construidos Evolutivamente mediante Avance por Voladizos Sucesivos," Tesis de Grado, Escuela Técnica Superior de Ingeniería, Universidad de Sevilla, Sevilla, 2011.

[15] H. R. Higuita, "Diseño de puentes de hormigón postensado construidos por voladizos sucesivos. Caso concreto de aplicación," Tesis de posgrado, Facultad Nacional de Minas, Universidad Nacional de Colombia, Medellín, 1998.

[16] Guide Specifications for Desing and Construction of Segmental Concrete Bridges \#2, American Association of State Highway and Transportation Officials, US, 1999.

[17] J. D. Honorio, "Conceptual design of long-span cantilever constructed concrete bridges," Tesis de Master, KTH, Estocolmo, 2007.
[18] M. G. Perdomo, "Estudio Comparativo de Puentes Construidos por Voladizos Sucesivos," Tesis de Master, Universitat Poliécnica de Catalunya, Barcelona, 2011.

[19] Structurae (2016). International Database for Civil and Structural Engineering. Berlin, Alemania. https://structurae.net/

[20] A. M. Agredo Chavez et al., "Evaluación de la rigidez a flexión de puentes de viga y losa en concreto presforzado a partir de pruebas de carga. Caso de estudio: Puente La Parroquia vía La Renta - San Vicente de Chucurí," UIS Ingenierías, vol. 15, no. 2, pp. 145-159, Jul-Dic 2016.

[21] MARTÍNEZ, Carolina, TORRES, Janneth y MEJÍA, Ruby. Evaluación de la toxicidad de un residuo industrial estabilizado/ solidificado con cemento portland. Rev.UIS.Ingenierías, 2014, vol.13, n.1, p.p 47-54. 